\title{
Hydrogels for tissue regeneration
}

\author{
Timothy E.L. Douglas \\ Engineering Department and Materials \\ Science Insitute, Lancaster University, \\ United Kingdom \\ t.douglas@lancaster.ac.uk
}

\begin{abstract}
Mineralization of hydrogels, or 3D polymer networks containing entrapped water, is considered desirable for applications in bone regeneration. We will present several strategies to mineralize hydrogels, including addition of preformed mineral particles, enzymatic mineralization and incorporation of calcium-binding substances.
\end{abstract}

\section{Keywords - hydrogel, mineralization, bone, composite}

\section{Motivation and aim}

Biomaterials for bone regeneration have predominantly been fabricated from inorganic substances such as various forms of calcium phosphate $(\mathrm{CaP})$, e.g.hydroxyapatite, tricalcium phosphate and brushite. $\mathrm{CaP}$ materials are mechanical stable and bioactive, i.e. they form a direct bone with surrounding bone tissue. However, such pure $\mathrm{CaP}$ materials have certain drawbacks. They are brittle, difficult to handle in granulate form and difficult to shape in block form. Furthermore, the incorporation of biologically active substances is not easy.

Hydrogels are highly hydrated three-dimensional polymer networks that are formed by crosslinking of polymer chains in solution. Hydrogels have been widely used as vehicles for drug delivery and are being used increasingly as biomaterials for tissue regeneration. As their main component is water, they have many advantages over pure inorganic materials. Firstly, the incorporation of water-soluble biologically active substances to promote tissue growth (e.g. growth factors) or to combat infection (e.g. antibiotics) is straightforward. Secondly, they are much less brittle. Thirdly, they can be implanted in a minimally invasive manner by injection, as they can undergo gelation, i.e. the transition from liquid to solid, after injection. However, their main disadvantage also stems from the fact that the mail component is water: hydrogels are mechanically weak. In order to combine the advantages of inorganic and hydrogel biomaterials, attention has recently been focused on the development of composites on the basis of mineralized hydrogels.

\section{Methods}

Several strategies have been tried [1]. The most common strategy is the addition of preformed inorganic particles to the polymer solution before gelation, after which the particles remain entrapped in the crosslinked polymer network. Ideally, the particles can be distributed homogeneously in the hydrogel. The gelation process can be induced by addition of inorganic particles. For example, the addition of bioactive glass particles to a solution of the anionic polysaccharide gellan gum results in hydrogel formation due to release of ions from the particles [2]. In other words, the particles serve as an "ion-delivery system" to provide homogeneous gelation. Another strategy is to promote precipitation of the inorganic phase in the hydrogel by increasing the concentration of ions. This can be achieved biomimetically using the enzyme alkaline phosphatase (ALP) which is responsible for the mineralization of bone tissue in vivo by cleaving phosphate ions from organophosphate and thus increasing the local phosphate concentration, which in turn promotes $\mathrm{CaP}$ precipitation [3]. Yet another strategy is the incorporation of calcium- or phosphate-binding molecules in the hydrogel, in order to increase local ion concentrations and promote $\mathrm{CaP}$ precipitation. Once such biomolecule is polydopamine, which binds calcium ions [4]. An added flexibility of mineralized hydrogels is the possibility of manipulation of either the hydrogel phase, or the inorganic phase, or both. For example, in the case of a hydrogel mineralized with $\mathrm{CaP}$, the inorganic phase may be modified by incorporation of magnesium in order to promote adhesion and proliferation of bone-forming cells [5], or by incorporation of zinc in order to endow antibacterial activity [6]. Alternatively, the hydrogel phase may be modified by incorporation of biologically active molecules such as polyphenols, which both bind calcium ions and exhibit antibacterial activity [7].

\section{Results}

All the aforementioned strategies have been successfully applied to mineralize hydrogels in previous work [1-8]. More recently research in our group has focussed on a novel material commonly used in food industry, namely whey protein isolate (WPI), which can be combined with particles to improve mechanical properties and cell behaviour [9-11].

\section{ACKNOWLEDGMENT}

FWO, Belgium and N8 Agrifood grant "Food2Bone", UK.

\section{REFERENCES}

[1] Gkioni K, et al. (2010) Mineralization of hydrogels for bone regeneration. Tissue Eng Part B Rev. 16(6): 577-85

[2] Douglas TE et al. (2014) Injectable self-gelling composites for bone tissue engineering based on gellan gum hydrogel enriched with different bioglasses. Biomed Mater. 9(4): 045014.

[3] Douglas TE, et al. (2012) Enzymatic mineralization of hydrogels for bone tissue engineering by incorporation of alkaline phosphatase. Macromol Biosci.12(8): 1077-89.

[4] Douglas TE, et al. (2014) Enzymatic mineralization of gellan gum hydrogel for bone tissue-engineering applications and its enhancement by polydopamine. J Tissue Eng Regen Med. 8(11): 906-18.

[5] Douglas TE, et al. (2016) Generation of composites for bone tissueengineering applications consisting of gellan gum hydrogels mineralized with calcium and magnesium phosphate phases by enzymatic means. J Tissue Eng Regen Med. 10(11): 938-954.

[6] Douglas TE, et al. (2017) Composites of gellan gum hydrogel enzymatically mineralized with calcium-zinc phosphate for bone regeneration with antibacterial activity. J Tissue Eng Regen Med. 11(5): 1610-1618.

[7] Douglas TE, et al. (2016). Biomed Mater. 11(4): 045015.

[8] Douglas TE, et al. (2018). J Tissue Eng Regen Med. 12(6): 1313-1326

[9] Dziadek M, et al. (2019) Novel multicomponent organic-inorganic $\mathrm{WPI} /$ gelatin/CaP hydrogel composites for bone tissue engineering. $\mathrm{J}$ Biomed Mater Res A. 107(11): 2479-2491.

[10] Dziadek M, et al. (2020). Mater Lett. 261: 127115

[11] Gupta D, et al. (2020) Novel naturally derived whey protein isolate and aragonite biocomposite hydrogels have potential for bone regeneration. Materials and Design. 188: 108408. 